\title{
ASYMPTOTICS OF HITCHIN'S METRIC ON THE HITCHIN SECTION
}

\author{
DAVID DUMAS AND ANDREW NEITZKE
}

\begin{abstract}
We consider Hitchin's hyperkähler metric $g$ on the moduli space $\mathcal{M}$ of degree zero SL(2)-Higgs bundles over a compact Riemann surface. It has been conjectured that, when one goes to infinity along a generic ray in $\mathcal{M}, g$ converges to an explicit "semiflat" metric $g^{\text {sf }}$, with an exponential rate of convergence. We show that this is indeed the case for the restriction of $g$ to the tangent bundle of the Hitchin section $\mathcal{B} \subset \mathcal{M}$.
\end{abstract}

\section{INTRODUCTION}

1.1. Summary. Fix a compact Riemann surface $C$. In [9] Hitchin studied the moduli space $\mathcal{M}$ of degree zero SL(2)-Higgs bundles on $C$, and showed in particular that $\mathcal{M}$ admits a canonically defined hyperkähler metric $g$.

In $[6,8]$ a new conjectural construction of $g$ was given. The full conjecture is complicated to state (see [13] for a review), but one of its consequences is a concrete picture of the generic asymptotics of $g$, as follows.

The non-compact space $\mathcal{M}$ is fibered over the space $\mathcal{B}$ of holomorphic quadratic differentials on $C$. We consider a path to infinity in $\mathcal{M}$, lying over a generic ray $\left\{t \phi_{0}\right\}_{t \in \mathbb{R}_{+}} \subset \mathcal{B}$, where $\phi_{0}$ has only simple zeroes. Along such a path, the prediction is that

$$
g=g^{\mathrm{sf}}+O\left(\mathrm{e}^{-4 \alpha t^{\frac{1}{2}}}\right)
$$

where $g^{\text {sf }}$ is the semiflat metric, given by a simple explicit formula (see §3.1), and $\alpha$ is any constant with $\alpha<M\left(\phi_{0}\right)$, where $M\left(\phi_{0}\right)$ is the length of the shortest saddle connection in the metric $\left|\phi_{0}\right|$ (see $\left.\S 3.2\right)$.

Very recently Mazzeo-Swoboda-Weiss-Witt [11] have shown that, along a generic ray, the difference $g-g^{\text {sf }}$ does decay at least polynomially in $t$. This work motivated us to wonder whether one could show directly that the decay is actually exponential. In this paper we show that this is indeed the case for the restriction of $g$ to the tangent bundle of a certain embedded copy of $\mathcal{B}$ inside $\mathcal{M}$, the Hitchin section: (1.1) holds there for any $\alpha<\frac{1}{2} M\left(\phi_{0}\right)$. (Unfortunately, we miss the conjectured sharp constant by a factor of 2.) The precise statement is given in Theorem 1 below.

1.2. The strategy. Points of $\mathcal{B}$ correspond to holomorphic quadratic differentials $\phi$ on $C$. Since these form a linear space, tangent vectors to $\mathcal{B}$ likewise correspond to holomorphic quadratic differentials $\dot{\phi}$. Given $(\phi, \dot{\phi}) \in T \mathcal{B}$, both $g_{\phi}(\dot{\phi}, \dot{\phi})$ and $g_{\phi}^{\text {sf }}(\dot{\phi}, \dot{\phi})$ arise as integrals over $C$ (which can be found in (4.23) and (4.24) below). The integrand in $g_{\phi}^{\text {sf }}$ is completely explicit, while the integrand in $g_{\phi}$ depends on the solutions of two elliptic scalar PDEs on the surface $C$. To prove (1.1) for some given $\alpha$, we need to show that these two integrals agree up to $O\left(\mathrm{e}^{-4 \alpha t^{\frac{1}{2}}}\right)$.

Date: March 27, 2018. (v1: February 20, 2018). 




Figure 1. A genus 2 surface $C$ equipped with a holomorphic quadratic differential $\phi_{0}$ which has 4 simple zeroes (orange crosses). The shortest saddle connection is shown in green; its length is $M\left(\phi_{0}\right)$. We have chosen $\alpha$ slightly smaller than $\frac{1}{2} M\left(\phi_{0}\right)$. $C_{\text {near }}$ is the union of 4 disks $D_{i}$ centered on the zeroes, all with the same radius $\alpha$. The complementary region $C_{\text {far }}$ is shaded.

To do this, we let $r_{0}(z)$ denote the $\left|\phi_{0}\right|$-distance from $z$ to the closest zero of $\phi_{0}$, and divide the surface $C$ into two regions, as illustrated in Figure 1:

- The "far" region $C_{\mathrm{far}}=\left\{z: r_{0}(z)>\alpha\right\}$. In this region we can show that the integrands agree to order $O\left(\mathrm{e}^{-4 \alpha t^{\frac{1}{2}}}\right)$ : indeed, we show that the difference $\delta$ of the integrands decays as $\delta=O\left(\mathrm{e}^{-\gamma t^{\frac{1}{2}} r_{0}(z)}\right)$ for any $\gamma<4$. This part of our analysis contains no big surprises, and is closely parallel to the analysis carried out by Mazzeo-Swoboda-Weiss-Witt in the more general setup of arbitrary SL(2)-Higgs bundles in [11]. (However, because we restrict to the Hitchin section $\mathcal{B} \subset \mathcal{M}$, our job is somewhat simpler: we only have to deal with scalar PDEs, and use more-or-less standard techniques. The specific estimates we use in this part are built on the work of Minsky in [12].)

- The "near" region $C_{\text {near }}=\left\{z: r_{0}(z) \leq \alpha\right\}$. This region looks more difficult because our estimates do not show that $\delta$ is close to zero here. The happy surprise - which was really the reason for writing this paper-is that when $\alpha<\frac{1}{2} M\left(\phi_{0}\right), \delta$ turns out to be close to an exact form that we can control, as follows. For any $\alpha<\frac{1}{2} M\left(\phi_{0}\right), C_{\text {near }}$ is a disjoint union of disks $D_{i}$ centered on the zeros of $\phi_{0}$. On each $D_{i}$ we show that $\delta=\mathrm{d} \beta_{i}+O\left(\mathrm{e}^{-4 \alpha t^{\frac{1}{2}}}\right)$, for a 1 -form $\beta_{i}$ which has the same decay property as $\delta$, namely $\beta_{i}=O\left(\mathrm{e}^{-\gamma t^{\frac{1}{2}}} r_{0}(z)\right)$. Thus $\beta_{i}$ is exponentially small on the boundary of $D_{i}$, and Stokes's theorem gives $\int_{D_{i}} \delta=\int_{\partial D_{i}} \beta_{i}+O\left(\mathrm{e}^{-4 \alpha t^{\frac{1}{2}}}\right)=O\left(\mathrm{e}^{-4 \alpha t^{\frac{1}{2}}}\right)$.

Combining these contributions we obtain the desired estimate $\int_{C} \delta=O\left(\mathrm{e}^{-4 \alpha t^{\frac{1}{2}}}\right)$.

1.3. Outline. We carry out the strategy described above as follows. In $\S \S 2-3$ we set up the background and notation, and state our main result precisely, as Theorem 1 . In $\S 4$ we derive integral expressions for the restrictions of $g$ and $g^{\text {sf }}$ to $T \mathcal{B}$. In $\S \S 5-7$ we develop the main PDE estimates we use to derive exponential decay. In $\S \S 8-9$ we construct the 1 -forms $\beta_{i}$ which we use in the "near" region. In $\S 10$ we put all this together to complete the proof of the main theorem.

1.4. Origin in experiment. This work was initially inspired by computer experiments (using programs developed by the authors, and building on work of the first author and Wolf in [4]) that seemed to show exponential decay of $g-g^{\text {sf }}$ in certain cases, despite the lack of an exponentially decaying bound on the integrand near the zeros of $\phi$. While these experiments were conducted in a slightly different setting - namely, meromorphic Higgs bundles on $\mathbb{C P}^{1}$ with a single pole - all of the essential features and challenges are present in both cases. The experimental results therefore suggested that some "cancellation" would occur in $C_{\text {near }}$. 
Further investigation of the integrand in this region led to the results of $\S \S 8-9$ below, and thus to the main theorem.

This experimental counterpart of this work is ongoing and will be the subject of a forthcoming paper and software release.

1.5. Outlook. It would be very desirable to understand how to extend Theorem 1 to Higgs bundles of higher rank, say SL( $N)$-Higgs bundles. There is a conjecture very similar to (1.1) in that case, but instead of the shortest saddle connection, it involves the lightest finite web as defined in [7]. While the analysis of $C_{\text {far }}$ should extend to this case using methods similar to those of [11], it is not clear how our approach to $C_{\text {near }}$ should be generalized.

Similarly, one would like to extend Theorem 1 to work on the full $\mathcal{M}$ instead of only $\mathcal{B} \subset \mathcal{M}$. The analysis of $C_{\text {far }}$ has already been done on the full $\mathcal{M}$ in [11], so again the issue is whether the analysis of $C_{\text {near }}$ can be extended.

In another direction, it would be desirable to improve Theorem 1 to show that the exponential estimate holds for all $\alpha<M\left(\phi_{0}\right)$ instead of just $\alpha<\frac{1}{2} M\left(\phi_{0}\right)$. However, this might require a new method; in our computation we meet several different corrections which are

naively of the same order $\mathrm{e}^{-2 M\left(\phi_{0}\right) t^{\frac{1}{2}}}$; one would need to find some mechanism by which these different corrections can cancel one another.

1.6. Acknowledgements. The authors thank Rafe Mazzeo, Jan Swoboda, Hartmut Weiss, and Michael Wolf for helpful discussions related to this work, and also thank the anonymous referee for a careful reading and helpful comments and corrections. The authors also gratefully acknowledge support from the U.S. National Science Foundation through individual grants DMS 1709877 (DD), DMS 1711692 (AN), and through the GEAR Network (DMS 1107452, 1107263, 1107367, "RNMS: GEometric structures And Representation varieties") which supported a conference where some of this work was conducted.

\section{BACKGROUND}

2.1. Higgs bundles. Recall that a stable SL(2)-Higgs bundle over $C$ of degree zero is a pair $(\mathcal{E}, \varphi)$ where

- $\mathcal{E}$ is a rank 2 holomorphic vector bundle over $C$, equipped with a trivialization of $\operatorname{det} \mathcal{E}$,

- $\varphi$ is a traceless holomorphic section of End $\mathcal{E} \otimes K_{C}$,

- all $\varphi$-invariant subbundles of $\mathcal{E}$ have negative degree.

There is a (coarse) moduli space $\mathcal{M}$ parameterizing stable $\mathrm{SL}(2)$-Higgs bundles over $C$ of degree zero $[9,10]$.

2.2. Harmonic metrics. For each stable $\operatorname{SL}(2)$-Higgs bundle $(\mathcal{E}, \varphi)$ of degree zero, it is shown in [9] that there is a distinguished unit-determinant Hermitian metric $h$ on $\mathcal{E}$, the harmonic metric. The metric $h$ is determined by solving an elliptic PDE: letting $D$ denote the Chern connection in $\mathcal{E}$, with curvature $F_{D} \in \Omega^{2}(\mathfrak{s u}(\mathcal{E}, h))$, and letting $\Phi=\varphi-\varphi^{\dagger} \in$ $\Omega^{1}(\mathfrak{s u}(\mathcal{E}, h))$ with $\varphi^{\dagger}$ the $h$-adjoint of $\varphi$, we require

$$
F_{D}-\frac{1}{2}[\Phi, \Phi]=0 .
$$

In this equation both $F_{D}$ and $\Phi$ depend on $h$. 
2.3. The hyperkähler metric. Now we recall Hitchin's hyperkähler metric $g$ on the moduli space $\mathcal{M}$. A beautiful description of this metric was given by Hitchin in [9] in terms of an infinite-dimensional hyperkähler quotient. In this paper we will not use the hyperkähler structure; all we need is a practical recipe for computing the metric. In this section we review that recipe.

Let $v$ be tangent to an arc in $\mathcal{M}$, and lift this arc to a family of Higgs bundles $\left(\mathcal{E}_{t}, \varphi_{t}\right)$, equipped with harmonic metrics $h_{t}$. Identify all the $\left(\mathcal{E}_{t}, h_{t}\right)$ with a fixed $C^{\infty} \mathrm{SU}(2)$-bundle $E$. Then we have a family of unitary connections $D_{t}$ on $E$ and 1 -forms $\Phi_{t} \in \Omega^{1}(\mathfrak{s u}(E))$ which for all $t$ satisfy (2.1). For brevity, let $D:=D_{0}$ and $\Phi:=\Phi_{0}$ denote these objects at $t=0$. Differentiating at $t=0$ we obtain a pair of 1 -forms

$$
(\dot{A}, \dot{\Phi})=\left(\left.\partial_{t} D_{t}\right|_{t=0},\left.\partial_{t} \Phi_{t}\right|_{t=0}\right) \in \Omega^{1}(\mathfrak{s u}(E))^{2} .
$$

Given $\alpha \in \Omega^{1}(\mathfrak{s u}(E))$ we define a nonnegative density $|\alpha|^{2}$ on $C$ by

$$
\left|\alpha_{x} \mathrm{~d} x+\alpha_{y} \mathrm{~d} y\right|^{2}=-\operatorname{Tr}\left(\alpha_{x}^{2}+\alpha_{y}^{2}\right) \mathrm{d} x \mathrm{~d} y .
$$

Here $z=x+\mathrm{i} y$ is a local conformal coordinate on $C$. In coordinate-independent terms, the density $|\alpha|^{2}$ corresponds (using the orientation of $C$ ) to the 2 -form $-\operatorname{Tr}(\alpha \wedge \star \alpha$ ), where $\star$ denotes the Hodge star operator on 1 -forms. Now we equip $\Omega^{1}(\mathfrak{s u}(E))^{2}$ with the $L^{2}$ metric

$$
\|(\dot{A}, \dot{\Phi})\|^{2}=\int_{C}\left(|\dot{A}|^{2}+|\dot{\Phi}|^{2}\right)
$$

Let $\rho: \Omega^{0}(\mathfrak{s u}(E)) \rightarrow \Omega^{1}(\mathfrak{s u}(E))^{2}$ be the linearized gauge map, defined by

$$
\rho(X)=\left(-\mathrm{d}_{D} X,[X, \Phi]\right) .
$$

We consider the orthogonal decomposition of $(\dot{A}, \dot{\Phi})$ relative to the image of $\rho$,

$$
(\dot{A}, \dot{\Phi})=(\dot{A}, \dot{\Phi})^{\|}+(\dot{A}, \dot{\Phi})^{\perp}
$$

with $(\dot{A}, \dot{\Phi})^{\|} \in \rho\left(\Omega^{0}(\mathfrak{s u}(E))\right.$ and $(\dot{A}, \dot{\Phi})^{\perp} \in \rho\left(\Omega^{0}(\mathfrak{s u}(E))^{\perp}\right.$. Hitchin's hyperkähler metric $g$ is

$$
g(v, v)=\left\|(\dot{A}, \dot{\Phi})^{\perp}\right\|^{2} .
$$

2.4. The Hitchin section. Fix a spin structure on the compact Riemann surface $C$. The spin structure determines a holomorphic line bundle $\mathcal{L}$ equipped with an isomorphism $\mathcal{L}^{2} \simeq$ $K_{C}$, and thus a rank 2 holomorphic vector bundle

$$
\mathcal{E}=\mathcal{L} \oplus \mathcal{L}^{-1} .
$$

This bundle has $\operatorname{det} \mathcal{E}=\mathcal{L} \otimes \mathcal{L}^{-1}$ which is canonically trivial. Let $\mathcal{B}$ be the space of holomorphic quadratic differentials on $C$,

$$
\mathcal{B}=H^{0}\left(C, K_{C}^{2}\right)
$$

For each $\phi \in \mathcal{B}$ there is a corresponding Higgs field,

$$
\varphi=\left(\begin{array}{cc}
0 & -\phi \\
1 & 0
\end{array}\right) \in H^{0}\left(C, \text { End } \mathcal{E} \otimes K_{C}\right) .
$$

The Higgs bundles $(\mathcal{E}, \varphi)$ are all stable, and thus determine a map $\iota: \mathcal{B} \rightarrow \mathcal{M}$. The image $\iota(\mathcal{B}) \subset \mathcal{M}$ is an embedded submanifold, the Hitchin section. ${ }^{1}$ Moreover, $\iota$ is a holomorphic map, with respect to the complex structure on $\mathcal{M}$ induced from its realization as moduli

\footnotetext{
${ }^{1}$ More precisely there are $2^{2 \times \operatorname{genus}(C)}$ Hitchin sections, corresponding to the equivalence classes of spin structures on $C$. All of our discussion applies to any of them.
} 
space of Higgs bundles (which is the complex structure denoted $I$ in [9]). Thus $\iota(\mathcal{B})$ is a complex submanifold of $\mathcal{M}$. From now on, by abuse of notation, we identify $\mathcal{B}$ with $\iota(\mathcal{B})$.

Our interest in this paper is in the restriction of the hyperkähler metric $g$ from the full $T \mathcal{M}$ to $T \mathcal{B}$. This restriction is a Kähler metric on $\mathcal{B}$, which we will also denote $g$.

\section{Metric estimate}

3.1. The semiflat metric. Let $\mathcal{B}^{\prime} \subset \mathcal{B}$ be the locus of quadratic differentials with only simple zeros, which is an open and dense set. On $\mathcal{B}^{\prime}$ we define an explicit Kähler metric $g^{\text {sf }}$ as follows. A tangent vector to $\mathcal{B}$ can be represented by a quadratic differential $\dot{\phi}$. We define

$$
g_{\phi}^{\mathrm{sf}}(\dot{\phi}, \dot{\phi})=2 \int_{C} \frac{|\dot{\phi}|^{2}}{|\phi|} .
$$

Note that the integrand on the right hand side is a smooth density on $C \backslash \phi^{-1}(0)$. The condition that $\phi \in \mathcal{B}^{\prime}$ implies that this integral is convergent.

We remark that $g^{\text {sf }}$ is a "(rigid) special Kähler" metric on $\mathcal{B}^{\prime}$ in the sense of [5]. It does not extend to a Riemannian metric on the full $\mathcal{B}$.

3.2. Threshold and radius. Any nonzero quadratic differential $\phi \in \mathcal{B}$ induces a flat metric $|\phi|$ on $C$, which is smooth except for conical singularities at the zeros of $\phi$. From now on we always use this metric to define geodesics and lengths on $C$, unless a different metric is explicitly referenced. A saddle connection of $\phi$ is a geodesic segment on $C$ which begins and ends on zeros of $\phi$ (not necessarily two distinct zeros), and which has no zeros of $\phi$ in its interior.

We define the threshold $M: \mathcal{B} \rightarrow \mathbb{R}_{\geqslant 0}$ by

$$
M(\phi)= \begin{cases}\text { the minimum length of a saddle connection of } \phi & \text { for } \phi \in \mathcal{B}^{\prime}, \\ 0 & \text { for } \phi \in \mathcal{B} \backslash \mathcal{B}^{\prime} .\end{cases}
$$

Then $M$ is continuous and has the homogeneity property

$$
M(t \phi)=t^{\frac{1}{2}} M(\phi), \quad t \in \mathbb{R}_{+} .
$$

The threshold measures the distance "between zeros" of $\phi$ (including the possibility of a segment between a zero and itself). In what follows it will also be important to consider the distance from an arbitrary point to the zeros of $\phi$. We define the radius function $r: C \rightarrow \mathbb{R}$ of $\phi$ by

$$
r(z)=d\left(z, \phi^{-1}(0)\right) .
$$

The main technical estimates that are used in the proof of Theorem 1 are all phrased in terms of bounds on various functions on $C$ in terms of the radius.

3.3. The estimate. Now we can state the main result of this paper:

Theorem 1. If $\phi_{0} \in \mathcal{B}^{\prime}$, and $\dot{\phi}$ is any holomorphic quadratic differential on $C$, then for any $\alpha<\frac{1}{2} M\left(\phi_{0}\right)$ we have

$$
\left|\left(g_{t \phi_{0}}-g_{t \phi_{0}}^{\mathrm{sf}}\right)(\dot{\phi}, \dot{\phi})\right|=O\left(\mathrm{e}^{-4 \alpha t^{\frac{1}{2}}}\|\dot{\phi}\|^{2}\right)
$$

as $t \rightarrow \infty$, where $\|\cdot\|$ denotes any norm on the vector space $\mathcal{B}$. Having fixed such a norm, the implicit multiplicative constant in (3.5) can be taken to depend only on $\alpha, M\left(\phi_{0}\right)$, and the genus of $C$. 


\section{Coordinate computations}

4.1. Self-duality equation and variation in coordinates. To set the stage for the proof of Theorem 1 we start by deriving local coordinate expressions for the self-duality equation (2.1) at a point $\phi \in \mathcal{B}$, and for its first variation in the direction of $(\dot{A}, \dot{\Phi})$ representing $\dot{\phi} \in T_{\phi} \mathcal{B}$.

In a local conformal coordinate $z=x+\mathrm{i} y$ on $C$ we write $\phi=P(z) \mathrm{d} z^{2}$ for a holomorphic function $P$. Let $\mathrm{d} z^{\frac{1}{2}}$ denote a local section of $\mathcal{L}$ satisfying $\mathrm{d} z^{\frac{1}{2}} \otimes \mathrm{d} z^{\frac{1}{2}}=\mathrm{d} z$; there are two such local sections, the choice of which will not matter in the sequel. Using the local trivialization of $\mathcal{E}=\mathcal{L} \oplus \mathcal{L}^{-1}$ given by the frame $\left(\mathrm{d} z^{\frac{1}{2}}, \mathrm{~d} z^{-\frac{1}{2}}\right)$, which we call the holomorphic gauge, we can write

$$
\begin{gathered}
\varphi=\left(\begin{array}{cc}
0 & -P \\
1 & 0
\end{array}\right) \mathrm{d} z, \quad h=\left(\begin{array}{cc}
\mathrm{e}^{-u} & 0 \\
0 & \mathrm{e}^{u}
\end{array}\right), \quad \varphi^{\dagger}=\left(\begin{array}{cc}
0 & \mathrm{e}^{2 u} \\
-\mathrm{e}^{-2 u} \bar{P} & 0
\end{array}\right) \mathrm{d} \bar{z}, \\
D=\mathrm{d}+A, \quad A=\left(\begin{array}{cc}
-\partial u & 0 \\
0 & \partial u
\end{array}\right) .
\end{gathered}
$$

This diagonal form for $h$ reflects that the splitting $\mathcal{L} \oplus \mathcal{L}^{-1}$ is orthogonal for the harmonic metric in this case [9, Theorem 11.2].

Then (2.1) reduces to a scalar equation for $u$,

$$
\Delta u-4\left(\mathrm{e}^{2 u}-\mathrm{e}^{-2 u}|P|^{2}\right)=0
$$

where $\Delta=4 \partial \bar{\partial}$ is the flat Laplacian.

In more invariant terms, (4.3) is an equation for the globally defined metric $\mathrm{e}^{2 u}|\mathrm{~d} z|^{2}$ on $C$. For Higgs bundles of this type, the Hermitian metric $h$, the Kähler metric $\mathrm{e}^{2 u}|\mathrm{~d} z|^{2}$, and the (local) scalar function $u$ all contain equivalent information. In most of what follows we work with $u$, which unlike $h$ and $\mathrm{e}^{2 u}|\mathrm{~d} z|^{2}$ is a coordinate-dependent quantity: Under a conformal change of coordinates $z \mapsto w$ it transforms as $u \mapsto u-\log \left|\frac{\mathrm{d} w}{\mathrm{~d} z}\right|$. We refer to objects with this transformation property as log densities. Note that the difference of two log densities is a function. Also, if $\phi=P \mathrm{~d} z^{2}$ is a quadratic differential, then $\frac{1}{2} \log |P|$ is a $\log$ density.

When considering the density $u$ on $C$ which corresponds to the unique harmonic metric on the Higgs bundle associated to $\phi \in \mathcal{B}$, we sometimes write $u(\phi)$ to emphasize its dependence on $\phi$, and to distinguish it from other local solutions to (4.3) on domains in $C$ or in the plane that we consider.

Next, we consider a variation $\dot{\phi} \in T_{\phi} \mathcal{B}$ expressed locally as $\dot{\phi}=\dot{P}(z) \mathrm{d} z^{2}$. Differentiating (4.3) we find that the corresponding first order variation $\dot{u}$, describing the infinitesimal change in $h$, satisfies the inhomogeneous linear equation

$$
\Delta \dot{u}-8 \dot{u}\left(\mathrm{e}^{2 u}+\mathrm{e}^{-2 u}|P|^{2}\right)+8 \mathrm{e}^{-2 u} \operatorname{Re}(P \dot{\bar{P}})=0 .
$$

Unlike $u, \dot{u}$ is a well-defined global function on $C$ (independent of the coordinate $z$ ). Since the operator $\Delta-8\left(\mathrm{e}^{2 u}+\mathrm{e}^{-2 u}|P|^{2}\right)$ is negative definite, (4.4) uniquely determines $\dot{u}$.

4.2. Unitary gauge. In preparation for calculating the $L^{2}$ inner product of variations it is more convenient to work in unitary gauge, expressing the Higgs field and connection relative to the frame $\left(\mathrm{e}^{\frac{1}{2} u} \mathrm{~d} z^{\frac{1}{2}}, \mathrm{e}^{-\frac{1}{2} u} \mathrm{~d} z^{-\frac{1}{2}}\right)$; then (4.1)-(4.2) become

$$
\varphi=\left(\begin{array}{cc}
0 & -\mathrm{e}^{-u} P \\
\mathrm{e}^{u} & 0
\end{array}\right) \mathrm{d} z, \quad \varphi^{\dagger}=\left(\begin{array}{cc}
0 & \mathrm{e}^{u} \\
-\mathrm{e}^{-u} \bar{P} & 0
\end{array}\right) \mathrm{d} \bar{z}, \quad A=\frac{\mathrm{i}}{2}\left(\begin{array}{cc}
\star \mathrm{d} u & 0 \\
0 & -\star \mathrm{d} u
\end{array}\right),
$$


with infinitesimal variations given by

$$
\begin{gathered}
\dot{\varphi}=\left(\begin{array}{cc}
0 & \mathrm{e}^{-u} P \dot{u}-\mathrm{e}^{-u} \dot{P} \\
\mathrm{e}^{u} \dot{u} & 0
\end{array}\right) \mathrm{d} z, \quad \dot{\varphi}^{\dagger}=\left(\begin{array}{cc}
0 & \mathrm{e}^{u} \dot{u} \\
\mathrm{e}^{-u} \bar{P} \dot{u}-\mathrm{e}^{-u} \dot{\bar{P}} & 0
\end{array}\right) \mathrm{d} \bar{z}, \\
\dot{A}=\frac{\mathrm{i}}{2}\left(\begin{array}{cc}
\star \mathrm{d} \dot{u} & 0 \\
0 & -\star \mathrm{d} \dot{u}
\end{array}\right)
\end{gathered}
$$

which of course gives a corresponding expression for $\dot{\Phi}=\dot{\varphi}-\dot{\varphi}^{\dagger}$.

4.3. Orthogonal decomposition. Let $(A, \Phi)$ be obtained from a solution of the self-duality equation (2.1). Define the linear map $\mu=\mu_{(A, \Phi)}: \Omega^{1}(\mathfrak{s u}(\mathcal{E}, h))^{2} \rightarrow \Omega^{2}(\mathfrak{s u}(\mathcal{E}, h))$ by

$$
\mu(\dot{A}, \dot{\Phi})=\mathrm{d}_{D} \star \dot{A}-[\dot{\Phi}, \star \Phi] .
$$

A variation $(\dot{A}, \dot{\Phi})$ is $L^{2}$-orthogonal to the image of the linearized gauge map $\rho$ if and only if it satisfies $\mu(\dot{A}, \dot{\Phi})=0$. We say that such a variation is in gauge.

For a general variation $(\dot{A}, \dot{\Phi})$, the orthogonal decomposition of $(2.6)$ is given by

$$
(\dot{A}, \dot{\Phi})^{\perp}=(\dot{A}, \dot{\Phi})-\rho(X)
$$

where $X \in \Omega^{0}(\mathfrak{s u}(E))$ satisfies $\mu(\rho(X))=\mu(\dot{A}, \dot{\Phi})$.

For the specific variation obtained in (4.6) we find that $\mathrm{d}_{D} \star \dot{A}=0$, and a straightforward calculation yields

$$
\begin{aligned}
Q:=\mu(\dot{A}, \dot{\Phi}) & =-[\dot{\Phi}, \star \Phi] \\
& =-2\left(\left[\varphi_{z}, \dot{\varphi}_{\bar{z}}^{\dagger}\right]+\left[\varphi_{\bar{z}}^{\dagger}, \dot{\varphi}_{z}\right]\right) \mathrm{d} x \mathrm{~d} y \\
& =-2 \mathrm{e}^{-2 u}(P \dot{\bar{P}}-\bar{P} \dot{P})\left(\begin{array}{cc}
1 & 0 \\
0 & -1
\end{array}\right) \mathrm{d} x \mathrm{~d} y .
\end{aligned}
$$

The computation of $(\dot{A}, \dot{\Phi})^{\perp}$ therefore reduces to solving

$$
\mu(\rho(X))=-\mathrm{d}_{D} \star \mathrm{d}_{D} X-[[X, \Phi], \star \Phi]=Q
$$

for $X$. Equation (4.10) implies in particular that $X$ is diagonal and traceless; thus we may write

$$
X=\frac{1}{2} \mathrm{i} v\left(\begin{array}{cc}
1 & 0 \\
0 & -1
\end{array}\right) .
$$

After so doing, (4.10) becomes a scalar equation for $v$,

$$
\Delta v-8 v\left(\mathrm{e}^{2 u}+\mathrm{e}^{-2 u}|P|^{2}\right)+8 \mathrm{e}^{-2 u} \operatorname{Im}(P \dot{\bar{P}})=0 .
$$

We note the striking similarity between (4.12) and (4.4); in fact, replacing $\dot{P} \rightarrow \mathrm{i} \dot{P}$ and $v \rightarrow-\dot{u}$ in (4.12) gives exactly (4.4). This suggests that we combine $\dot{u}$ (the metric variation) and $v$ (the infinitesimal gauge transformation to put the tangent vector in gauge) into the single complex function

$$
F=\dot{u}-\mathrm{i} v,
$$

which we call the complex variation, which then satisfies the inhomogeneous linear equation

$$
\left(\Delta-8\left(\mathrm{e}^{2 u}+\mathrm{e}^{-2 u}|P|^{2}\right)\right) F+8 \mathrm{e}^{-2 u} \bar{P} \dot{P}=0 .
$$

As with (4.4) above, when working on the entire compact surface $C$ the equation (4.14) uniquely determines the complex function $F$. We write $F(\phi, \dot{\phi})$ for this unique global solution 
determined by $(\phi, \dot{\phi}) \in T_{\phi} \mathcal{B}$ when it is necessary to distinguish it from other local solutions of the same equation.

4.4. Calculating the norm. Using the calculations above we can now determine an explicit integral expression for $g_{\phi}(\dot{\phi}, \dot{\phi})$ in terms of $P, \dot{P}, u$, and $F$.

The first step is to calculate $\rho(X)$ in unitary gauge. We find $\rho(X)=(B, \Psi)$ where

$$
B=\frac{\mathrm{i}}{2}\left(\begin{array}{cc}
\mathrm{d} v & 0 \\
0 & -\mathrm{d} v
\end{array}\right), \quad \Psi=\psi-\psi^{\dagger}, \quad \psi=\mathrm{i} v\left(\begin{array}{cc}
0 & \mathrm{e}^{-u} P \\
\mathrm{e}^{u} & 0
\end{array}\right) \mathrm{d} z .
$$

Now $(\dot{A}, \dot{\Phi})^{\perp}=(\dot{A}, \dot{\Phi})-(B, \Psi)$ is orthogonal to $(B, \Psi)$, hence the hyperkähler norm of the associated tangent vector to the moduli space $\mathcal{M}$ is

$$
g_{\phi}(\dot{\phi}, \dot{\phi})=\left\|(\dot{A}, \dot{\Phi})^{\perp}\right\|^{2}=\|(\dot{A}, \dot{\Phi})\|^{2}-\|(B, \Psi)\|^{2} .
$$

Now we need only to substitute the expressions for $(\dot{A}, \dot{\Phi})$ from $(4.6)$ and $(B, \Psi)$ from (4.15) and simplify. Two observations will be useful in doing this. First, if $\Xi=\xi-\xi^{\dagger} \in \Omega^{1}(\mathfrak{s u}(\mathcal{E}))$ where $\xi$ is expressed in unitary gauge as $\xi=f(z) \mathrm{d} z$ for $f$ a matrix-valued function, then

$$
|\Xi|^{2}=4 \operatorname{tr}\left(f \bar{f}^{t}\right) \mathrm{d} x \mathrm{~d} y \text {. }
$$

Second, if $\beta=\left(\begin{array}{cc}\theta & 0 \\ 0 & -\theta\end{array}\right)$, with $\theta$ a scalar 1 -form, then we have

$$
|\beta|^{2}=|\star \beta|^{2}=2 \theta \wedge \star \theta .
$$

Using (4.17) to simplify $|\dot{\Phi}|^{2}$ and (4.18) to simplify $|\dot{A}|^{2}$, we find ${ }^{2}$

$$
\|(\dot{A}, \dot{\Phi})\|^{2}=\int_{C}|\dot{A}|^{2}+|\dot{\Phi}|^{2}=\frac{1}{2}\|\mathrm{~d} \dot{u}\|^{2}+\int_{C} 4\left(\mathrm{e}^{2 u} \dot{u}^{2}+\mathrm{e}^{-2 u}|P \dot{u}-\dot{P}|^{2}\right) \mathrm{d} x \mathrm{~d} y,
$$

where $\|\theta\|^{2}=\int_{C} \theta \wedge \star \theta$. Proceeding similarly for $\|(B, \Psi)\|^{2}$ using (4.15), we have

$$
\|(B, \Psi)\|^{2}=\frac{1}{2}\|\mathrm{~d} v\|^{2}+\int_{C}\left(4\left(\mathrm{e}^{2 u}+\mathrm{e}^{-2 u}|P|^{2}\right) v^{2}\right) \mathrm{d} x \mathrm{~d} y .
$$

Subtracting (4.20) from (4.19) we obtain

$$
g_{\phi}(\dot{\phi}, \dot{\phi})=\int_{C}\left(4 \mathrm{e}^{2 u} \dot{u}^{2}+4 \mathrm{e}^{-2 u}|P \dot{u}-\dot{P}|^{2}-4\left(\mathrm{e}^{2 u}+\mathrm{e}^{-2 u}|P|^{2}\right) v^{2}\right) \mathrm{d} x \mathrm{~d} y+\frac{1}{2}\|\mathrm{~d} \dot{u}\|^{2}-\frac{1}{2}\|\mathrm{~d} v\|^{2} \text {. }
$$

Next we integrate by parts on $C$ to replace $\|\mathrm{d} \dot{u}\|^{2}$ and $\|\mathrm{d} v\|^{2}$ by $-\int_{C}(\dot{u} \Delta \dot{u}) \mathrm{d} x \mathrm{~d} y$ and $-\int_{C}(v \Delta v) \mathrm{d} x \mathrm{~d} y$ respectively, and substitute for $\Delta \dot{u}$ and $\Delta v$ using the differential equations (4.3) and (4.12). A few terms cancel and we are left with

$$
g_{\phi}(\dot{\phi}, \dot{\phi})=\int_{C}\left(4 \mathrm{e}^{-2 u}|\dot{P}|^{2}-4 \mathrm{e}^{-2 u} \dot{u} \operatorname{Re}(P \dot{\bar{P}})-4 \mathrm{e}^{-2 u} v \operatorname{Im}(P \dot{\bar{P}})\right) \mathrm{d} x \mathrm{~d} y
$$

or more compactly,

$$
g_{\phi}(\dot{\phi}, \dot{\phi})=\int_{C} 4 \mathrm{e}^{-2 u}\left(|\dot{P}|^{2}-\operatorname{Re}(F P \dot{\bar{P}})\right) \mathrm{d} x \mathrm{~d} y
$$

As a reassuring consistency check, note that $g_{\phi}$ is indeed a Hermitian metric, i.e. $g_{\phi}(\mathrm{i} \dot{\phi}, \mathrm{i} \dot{\phi})=$ $g_{\phi}(\dot{\phi}, \dot{\phi})$ : one sees this easily from (4.23), since changing $\dot{\phi} \rightarrow \mathrm{i} \dot{\phi}$ leads to $F \rightarrow \mathrm{i} F$ and $\dot{P} \rightarrow \mathrm{i} \dot{P}$.

\footnotetext{
${ }^{2}$ Abusing notation, we often write integrals over $C$ with the integrand expressed in a local coordinate and frame for $\mathcal{E}$.
} 
The same is not true of (4.19) by itself: it holds only once we subtract the pure gauge part (4.20).

To sum up the results of this section, and restate the formula (3.1) for $g^{\text {sf }}$ in the same local coordinates, we have:

Theorem 2. For any quadratic differential $\phi \in \mathcal{B}^{\prime}$ and tangent vector $\dot{\phi} \in T_{\phi} \mathcal{B}^{\prime}=\mathcal{B}$ with respective coordinate expressions $\phi=P(z) \mathrm{d} z^{2}$ and $\dot{\phi}=\dot{P}(z) \mathrm{d} z^{2}$, the norm of $\dot{\phi}$ in the hyperkähler metric $g$ is given by (4.23), where $u$ and $F$ are the solutions of (4.3) and (4.14). The norm of the same tangent vector in the semiflat metric $g^{\text {sf }}$ is

$$
g_{\phi}^{\mathrm{sf}}(\dot{\phi}, \dot{\phi})=\int_{C} 2|P|^{-1}|\dot{P}|^{2} \mathrm{~d} x \mathrm{~d} y .
$$

The goal of the next three sections is to gain some control over the integral expressions (4.23) and (4.24) by studying the behavior of the functions $u$ and $F$. We will see that these functions are well-approximated by

$$
\begin{aligned}
u & \approx \frac{1}{2} \log |P|, \\
F & \approx \frac{1}{2} \frac{\dot{P}}{P},
\end{aligned}
$$

at points that are not too close to the zeros of $\phi$. It is easy to check that substituting these approximations directly into (4.23) yields exactly the semiflat integral (4.24). Bounding the difference $g-g^{\text {sf }}$ thus reduces to understanding the error in the approximations.

\section{EXPONENTIAL DECAY PRINCIPLE}

We now develop a criterion for solutions to certain elliptic PDE on regions in the plane to decay exponentially fast as we move away from the boundary of the region. The method is standard - combining the maximum principle with the known behavior of the eigenfunctions of the Laplacian - and the results in this section are surely not new. A similar method was used in [12], for example, to derive the exponential decay results for (4.3) that we will generalize in $\S 6$.

Theorem 3. Let $\Omega=\{|z|<R\}$ be a disk in $\mathbb{C}$, and for $z \in \Omega$ let $\rho(z)=d(z, \partial \Omega)=R-|z|$ denote the distance to the boundary of this disk. Suppose that $w \in C^{2}(\Omega) \cap C^{0}(\bar{\Omega})$ satisfies

$$
\left(\Delta-k^{2}\right) w=g
$$

where $k, g \in C^{0}(\bar{\Omega}), k \geqslant 4$, and suppose that for every $\gamma<4$ there exists a constant $A(\gamma)$ such that $g$ obeys the exponential decay condition

$$
|g|<A(\gamma) \mathrm{e}^{-\gamma \rho} .
$$

Then, for any $\gamma<4$, there exist constants $K(\gamma)$ and $A^{\prime}(\gamma)$, such that $w$ obeys the exponential decay condition

$$
|w|<K(\gamma)\left(M+A^{\prime}(\gamma)\right) \mathrm{e}^{-\gamma \rho},
$$

where $M=\sup _{\partial \Omega}|w|$. Moreover, given any $\gamma^{\prime}>\gamma, A^{\prime}(\gamma)$ can be chosen to be equal to $A\left(\gamma^{\prime}\right)$.

The proof will rely on the following maximum principle. 
Lemma 4. Let $\Omega$ be a bounded region in $\mathbb{C}$, and let $w, v \in C^{2}(\Omega) \cap C^{0}(\bar{\Omega})$ with $v \geqslant 0$. Suppose $w$ satisfies

$$
\left(\Delta-k^{2}\right) w=g
$$

and that $v$ satisfies

$$
\left(\Delta-\underline{k}^{2}\right) v=-\bar{g}
$$

where $k, g, \underline{k}, \bar{g} \in C^{0}(\bar{\Omega})$ are functions such that

$$
k \geqslant \underline{k}>0 \text { and }|g| \leqslant \bar{g} .
$$

If $|w| \leqslant v$ on $\partial \Omega$, then $|w| \leqslant v$ on $\bar{\Omega}$.

Proof. First we claim $w \leqslant v$, or equivalently that $v-w \geqslant 0$ on $\Omega$. By compactness of $\bar{\Omega}$, the function $v-w$ achieves its minimum at a point $p$, and it suffices to show that the minimum value is nonnegative. If $p \in \partial \Omega$ then this is true by the hypothesis that $|w| \leqslant v$ on $\partial \Omega$. If $w(p) \leqslant 0$ then $(v-w)(p) \geqslant 0$ because $v$ is everywhere nonnegative. Thus the remaining case is that $w(p)>0$ and $p$ is an interior local minimum of $v-w$, hence $\Delta(v-w)(p) \geqslant 0$. Then we find

$$
\begin{aligned}
0 \leqslant \Delta(v-w)(p) & =\underline{k}(p)^{2} v(p)-k(p)^{2} w(p)-\bar{g}(p)-g(p) & \text { using }(5.4),(5.5) \\
& \leqslant \underline{k}^{2}(p)(v-w)(p)-\bar{g}(p)-g(p) & \text { because } w(p)>0 \text { and } k \geqslant \underline{k} \\
& \leqslant \underline{k}^{2}(p)(v-w)(p) & \text { because } \bar{g}+g \geqslant 0
\end{aligned}
$$

Since $\underline{k}>0$ this shows $(v-w)(p) \geqslant 0$ as required.

To complete the proof we must also show that $-w \leqslant v$. However, this follows by applying the argument above to the function $w^{\prime}=-w$, which satisfies $\left(\Delta-k^{2}\right) w^{\prime}=g^{\prime}$, where $g^{\prime}=-g$. Since $\left|w^{\prime}\right|=|w| \leqslant v$ on $\partial \Omega$ and $\left|g^{\prime}\right|=|g| \leqslant \bar{g}$, the necessary hypotheses still hold in this case.

In the proof of Theorem 3 we will use Lemma 4 to reduce to the case where $k$ is constant and where $g$ and $w$ are both radially symmetric eigenfunctions of the Laplacian. In preparation for doing this, we recall the properties of those eigenfunctions and relate them to the exponential decay behavior under consideration.

The modified Bessel function of the first kind $I_{0}$ is the unique positive, even, smooth function on $\mathbb{R}$ such that

$$
\Delta I_{0}(|z|)=I_{0}(|z|)
$$

and $I_{0}(0)=1$. Thus the function $I_{0}(c|z|) / I_{0}(c R)$ is the solution to the Dirichlet problem for $\left(\Delta-c^{2}\right)$ on the disk $|z|<R$ with unit boundary values.

The function $I_{0}(x)$ satisfies (see e.g. [1, Section 9.7.1])

$$
I_{0}(x) \sim(2 \pi x)^{-\frac{1}{2}} \mathrm{e}^{x}
$$

where $f \sim g$ means that $f(x) / g(x) \rightarrow 1$ as $x \rightarrow \infty$. It follows that, if a function $f$ satisfies an exponential decay condition

$$
f<A \mathrm{e}^{-\gamma \rho}
$$

for some $\gamma>0$, then for any $\tilde{\gamma}<\gamma$ we have

$$
f<\tilde{A} \frac{I_{0}(\tilde{\gamma}|z|)}{I_{0}(\tilde{\gamma} R)}
$$


for some $\tilde{A}(A, \gamma, \tilde{\gamma})$ linear in $A$. Conversely, if we have (5.10) and $\gamma \leq \tilde{\gamma}$ then we get (5.9) for some $A(\tilde{A}, \gamma, \tilde{\gamma})$ linear in $\tilde{A}^{3}$

Proof of Theorem 3. Suppose we are given constants $\gamma<\gamma^{\prime}<4$. The function $g$ obeys

$$
|g|<A\left(\gamma^{\prime}\right) \mathrm{e}^{-\gamma^{\prime} \rho}
$$

and thus

$$
|g|<\tilde{A} \frac{I_{0}(\gamma|z|)}{I_{0}(\gamma R)}
$$

for some $\tilde{A}\left(A, \gamma, \gamma^{\prime}\right)$ linear in $A=A\left(\gamma^{\prime}\right)$. For $w$ satisfying (5.1) and $g$ satisfying (5.12) we will show that

$$
|w|<\tilde{K}(M+\tilde{A}) \frac{I_{0}(\gamma|z|)}{I_{0}(\gamma R)}
$$

for some $\tilde{K}(\gamma)$. Once this is achieved we can pass back from (5.13) to the desired (5.3) using the exponential bound on $I_{0}$ discussed above. Moreover, since $\tilde{A}$ depends linearly on $A\left(\gamma^{\prime}\right)$, we can choose $A^{\prime}=A$ in (5.3), at the cost of possibly rescaling $\tilde{K}$.

Define

$$
v=B I_{0}(\gamma|z|)
$$

for a constant $B>0$. Note that $\left(\Delta-\gamma^{2}\right) v=0$. We will determine a value of $B$ so that Lemma 4 can be applied to $v$ and $w$ on $\Omega$. Specifically, we must ensure that:

(i) $|w| \leqslant v$ on $\partial \Omega$ and

(ii) $(\Delta-16) v \leqslant-|g|$ on $\Omega$,

so that, in the notation of Lemma 4 , we can take $\bar{g}=-(\Delta-16) v$ and $\underline{k}=4$.

First we consider (i). The function $v$ is constant on $\partial \Omega$ and equal to $B I_{0}(\gamma R)$. Since $M=\sup _{\partial \Omega}|w|$, it suffices to choose

$$
B \geqslant \frac{M}{I_{0}(\gamma R)}
$$

Now we turn to (ii). We have

$$
(\Delta-16) v=-\left(16-\gamma^{2}\right) v
$$

which we have written in this way to emphasize that $\left(16-\gamma^{2}\right)>0$. With the given bound (5.12) on $|g|$, the desired inequality (ii) follows if

$$
B I_{0}(\gamma|z|)\left(16-\gamma^{2}\right) \geqslant \tilde{A} \frac{I_{0}(\gamma|z|)}{I_{0}(\gamma R)},
$$

or equivalently

$$
B \geqslant \frac{\tilde{A}}{16-\gamma^{2}} \frac{1}{I_{0}(\gamma R)} .
$$

Using (5.15) and (5.18) it is easy to verify that

$$
B=\max \left(1, \frac{1}{16-\gamma^{2}}\right) \cdot(M+\tilde{A}) \cdot \frac{1}{I_{0}(\gamma R)}
$$

\footnotetext{
${ }^{3}$ Adjusting the constants when converting between exponential and Bessel bounds is necessary due to the $x^{-\frac{1}{2}}$ factor in the expansion of $I_{0}$, with the relevant observation being that $x^{\frac{1}{2}} \mathrm{e}^{-\gamma x}=O\left(\mathrm{e}^{-\tilde{\gamma} x}\right)$ for all $\tilde{\gamma}<\gamma$ whereas of course $x^{\frac{1}{2}} \mathrm{e}^{-\gamma x} \neq O\left(\mathrm{e}^{-\gamma x}\right)$.
} 
satisfies both conditions (i)-(ii), and then by Lemma 4 we find $|w| \leqslant v$ on $D$, which is the desired bound (5.13) with

$$
\tilde{K}=\max \left(1, \frac{1}{16-\gamma^{2}}\right)
$$

\section{Estimates FOR THE DENSity $u$}

As in Section 4 above, let $u=u(\phi)$ be the solution of the self-duality equation (4.3) on the compact Riemann surface $C$ for a given holomorphic quadratic differential $\phi \in \mathcal{B}$ expressed locally as $\phi=P(z) \mathrm{d} z^{2}$. It was shown by Minsky in [12] (see also [14, Lemma 2.2]) that $u$ is approximated by $\frac{1}{2} \log |P|$ up to an error that decays exponentially in the distance from the zeros of $\phi$. Building on Minsky's results (and following a similar outline to [3, Section 5.4]), we establish the following estimate which gives a slightly faster exponential decay rate:

Theorem 5. Fix $\phi \in \mathcal{B}$ and assume $M(\phi)>1$. For any $\gamma<4$, there exist constants $A(\gamma)$ and $b(\gamma)$ such that the density $u=u(\phi)$ satisfies

$$
\left|u(z)-\frac{1}{2} \log \right| P(z)||<A(\gamma) \mathrm{e}^{-\gamma r(z)}
$$

for all $z \in C$ with $r(z)>b(\gamma)$. The constants $A(\gamma)$ and $b(\gamma)$ can be taken to depend only on $\gamma$ and the topological type of $C$.

Furthermore, under the same hypotheses we have the $C^{1}$ estimate

$$
\left|\nabla_{\phi}\left(u-\frac{1}{2} \log |P|\right)(z)\right|_{\phi}<A(\gamma) \mathrm{e}^{-\gamma r(z)}
$$

where $\nabla_{\phi}$ and $|v|_{\phi}$ denote, respectively, the gradient and the norm of a tangent vector with respect to the metric $|\phi|$.

To prove this, we will first establish some rough bounds on $u$. These will allow us to apply Theorem 3 to the equation satisfied by $u-\frac{1}{2} \log |P|$.

6.1. Rough bounds. Let $\mathrm{e}^{2 \sigma}|\mathrm{d} z|^{2}$ be the Poincaré metric on $C$ of constant (Gaussian) curvature -4 . In general, the Gaussian curvature of a metric $\mathrm{e}^{2 u}|\mathrm{~d} z|^{2}$ is given by $K=$ $-\mathrm{e}^{-2 u} \Delta u$ (see e.g. [2, Section 1.5]); therefore, the equation $K=-4$ satisfied by the Poincaré metric becomes

$$
\Delta \sigma=4 \mathrm{e}^{2 \sigma}
$$

which is equation (4.3) with $\phi=0$.

Now for the solution $u=u(\phi)$ of (4.3) associated to a general quadratic differential $\phi=P \mathrm{~d} z^{2}$, we have the following lower bounds in terms of $\sigma$ and $P$ :

Lemma 6. We have $u-\sigma \geqslant 0$ everywhere on $C$, and $u-\frac{1}{2} \log |P| \geqslant 0$ on $C \backslash \phi^{-1}(0)$.

Proof. Using (4.3) and (6.3) we have

$$
\Delta(u-\sigma)=4\left(\mathrm{e}^{2 u}-\mathrm{e}^{2 \sigma}-\mathrm{e}^{-2 u}|P|^{2}\right) \leqslant 4\left(\mathrm{e}^{2 u}-\mathrm{e}^{2 \sigma}\right) .
$$

At a minimum of $u-\sigma$, we have $\Delta(u-\sigma) \geqslant 0$ and so by (6.4) we find $\mathrm{e}^{2 u}-\mathrm{e}^{2 \sigma} \geqslant 0$ there. Thus $u-\sigma \geqslant 0$ at the minimum, hence everywhere on $C$. 
The lower bound on $u-\frac{1}{2} \log |P|$ is similar. Using that $\frac{1}{2} \log |P|$ is harmonic on $C \backslash \phi^{-1}(0)$, we find that

$$
\Delta\left(u-\frac{1}{2} \log |P|\right)=4\left(\mathrm{e}^{2 u}-\mathrm{e}^{-2 u}|P|^{2}\right) .
$$

Since $\frac{1}{2} \log |P(z)| \rightarrow-\infty$ as $z$ approaches a zero of $\phi$, while $u$ is smooth on the entire surface $C$, the difference $u-\frac{1}{2} \log |P|$ has a minimum on $C \backslash \phi^{-1}(0)$. At such a minimum we have $\Delta\left(u-\frac{1}{2} \log |P|\right) \geqslant 0$, which gives $\mathrm{e}^{2 u}-\mathrm{e}^{-2 u}|P|^{2} \geqslant 0$. This implies $u-\frac{1}{2} \log |P| \geqslant 0$ at a minimum, and thus everywhere.

Complementing these lower bounds on $u$, we have the following rough comparison to the singular flat metric $|\phi|$. Recall that the radius $r(z)$ is the distance from $z$ to $\phi^{-1}(0)$ with respect to the metric $|\phi|$.

Lemma 7 (Minsky [12, Lemma 3.2]). Fix $\phi \in \mathcal{B}$ and assume $M(\phi)>1$. Let $z \in C$ be a point with $r(z) \geqslant 1$. Then $u(z)-\frac{1}{2} \log |P(z)| \leqslant M$ where $M$ is a constant depending only on the topological type of $C$.

Note that Minsky's bound is more general, giving an upper bound at any point $z$ depending only on the topological type of $C$ and on the $|\phi|$-radius $R$ of an embedded disk centered at $z$ that contains no zeros of $\phi$. The hypotheses of the lemma above give such a disk of definite radius (in fact, one can take $R=\frac{1}{2}$ ), resulting in the bound stated above that only depends on the topological type.

\subsection{Exponential bounds.}

Proof of Theorem 5. We start with the $C^{0}$ bound (6.1). Consider a local coordinate $\zeta$ about $z$ in which $\phi=\mathrm{d} \zeta^{2}$. Allowing this coordinate chart to be immersed, rather than embedded, we can take it to be defined on $|\zeta|<r(z)$ with $z$ corresponding to $\zeta=0$. While the boundary of this disk touches the zero set of $\phi$ (by definition of $r$ ), if we consider $D=\{|\zeta|<r(z)-1\}$ then the image of this disk in $C$ consists of points satisfying the hypotheses of Lemma 7. Therefore, by Lemma 6 and Lemma 7 we have $0 \leqslant u(\zeta) \leqslant M$ for all $\zeta \in D$.

In this coordinate system we have $P(\zeta) \equiv 1$ and thus $(6.5)$ becomes

$$
\Delta u=4 \mathrm{e}^{2 u}-4 \mathrm{e}^{-2 u}=8 \sinh (2 u) .
$$

Similarly, in this coordinate the difference $\left|u-\frac{1}{2} \log \right| P||$ reduces to $|u|$.

The function $8 \sinh (2 x) / x$ has a removable singularity at $x=0$; let $f$ denote its extension to a smooth function on $\mathbb{R}$, which satisfies $f(x) \geqslant 16$ for $x \geqslant 0$. Since $u \geqslant 0$ we can rewrite the equation above as

$$
\left(\Delta-k^{2}\right) u=0
$$

where $k=\sqrt{f(u)}$, and thus $k \geqslant 4$. Now Theorem 3 applies to $u$ on $D$ with $g \equiv 0$ and $\rho=r(z)-1$, giving

$$
|u| \leqslant A(\gamma) \mathrm{e}^{-\gamma(r(z)-1)}
$$

for all $\gamma<4$. Absorbing the $\mathrm{e}^{\gamma}$ factor into the multiplicative constant we obtain the desired bound (6.1) in terms of $\mathrm{e}^{-\gamma r(z)}$.

Given this $C^{0}$ bound, the corresponding $C^{1}$ bound (6.2) follows by standard elliptic theory applied to (6.6), as shown in e.g. [3, Corollary 5.10]. 


\section{Estimates FOR THE COMPLEX VARIATION $F$}

Next we turn to the complex variation $F=F(\phi, \dot{\phi})$ associated to $\phi \in \mathcal{B}^{\prime}$ and $\dot{\phi} \in T_{\phi} \mathcal{B}$. We will see that this function is exponentially close to $\frac{1}{2} \frac{\dot{\phi}}{\phi}=\frac{1}{2} \frac{\dot{P}}{P}$. Specifically, we have:

Theorem 8. Fix $\phi \in \mathcal{B}$ and assume $M(\phi)>1$. Also fix $\dot{\phi}$. For any $\gamma<4$, there exist constants $A(\gamma)$ and $b(\gamma)$ such that the function $F(\phi, \dot{\phi})$ satisfies

$$
\left|F-\frac{1}{2} \frac{\dot{\phi}}{\phi}\right|<A(\gamma)\|\dot{\phi}\| \mathrm{e}^{-\gamma r(z)}
$$

for all $z \in C$ with $r(z)>b(\gamma)$.

To prove this, we proceed as in $\S 6$, first deriving some rough bounds, and then improving them to exponential bounds using Theorem 3.

7.1. Rough bounds. We begin with some notation related to metrics on $C$. If $\eta$ is a $\log$ density on $C$, with associated Kähler metric $\mathrm{e}^{2 \eta}\left|\mathrm{d} z^{2}\right|$, and if $\phi \in \mathcal{B}$ has local expression $\phi=P \mathrm{~d} z^{2}$, we denote by

$$
|\phi|_{\eta}=\mathrm{e}^{-2 \eta}|P|: C \rightarrow \mathbb{R}
$$

the pointwise norm function of $\phi$ with respect to this metric, and by

$$
\|\phi\|_{\eta}=\sup _{C}|\phi|_{\eta}
$$

the associated sup-norm. Finally, we let

$$
\Delta_{\eta}=\mathrm{e}^{-2 \eta} \Delta
$$

denote the Laplace-Beltrami operator of the metric $\mathrm{e}^{2 \eta}\left|\mathrm{d} z^{2}\right|$.

Recall from $\S 6$ that $\sigma$ denotes the density of the Poincaré metric on $C$ of curvature -4 .

Lemma 9. The complex variation $F$ satisfies $\sup |F| \leqslant\|\dot{\phi}\|_{\sigma}$.

Proof. Rewriting (4.14) in terms of the Laplace-Beltrami operator $\Delta_{u}$ it becomes:

$$
\left(\Delta_{u}-K\right) F=-G
$$

where

$$
\begin{aligned}
& K=8\left(1+|\phi|_{u}^{2}\right), \\
& G=\frac{8 \bar{\phi} \dot{\phi}}{\mathrm{e}^{4 u}|\mathrm{~d} z|^{4}} .
\end{aligned}
$$

Note that $K>0$ and that $G$ is a well-defined complex scalar function on $C$ which satisfies

$$
|G|=8|\phi|_{u}|\dot{\phi}|_{u}
$$

Considering a maximum and a minimum of each of the real and imaginary parts of $F$, which exist by compactness, we find from (7.5) that

$$
\sup |F| \leqslant \sup 2 K^{-1}|G|
$$

and we have

$$
K^{-1}|G|=|\dot{\phi}|_{u}\left(\frac{|\phi|_{u}}{1+|\phi|_{u}^{2}}\right) \leqslant \frac{1}{2}|\dot{\phi}|_{u}
$$


Finally, by Lemma 6 , we have $u \geqslant \sigma$. Therefore $|\dot{\phi}|_{u} \leqslant|\dot{\phi}|_{\sigma}$ and

$$
\sup |F| \leqslant \sup 2 K^{-1}|G| \leqslant \sup |\dot{\phi}|_{\sigma}=\|\dot{\phi}\|_{\sigma} .
$$

We will also need the following lower bound on the pointwise norm $|\phi|_{\sigma}$. Recall $r(z)$ denotes the $|\phi|$-distance from $z$ to $\phi^{-1}(0)$.

Lemma 10. Let $\phi \in \mathcal{B}^{\prime}$ and suppose $\|\phi\|_{\sigma} \geqslant 1$. There exists a constant $\delta$ depending only on the ray $\mathbb{R}_{+} \phi$ with the following property: If $z \in C$ satisfies $r(z)>1$, then $|\phi|_{\sigma}(z) \geqslant \delta$.

Proof. Let $Z=\phi^{-1}(0)$. First suppose that $\|\phi\|_{\sigma}=1$. For any positive radius $r_{0}$, a uniform lower bound on $|\phi|_{\sigma}(z)$ for $z$ with $r(z) \geqslant r_{0}$ follows immediately from compactness of $C$ and of the unit ball in $\mathcal{B}$.

Using that $d_{t \phi}=t^{1 / 2} d_{\phi}$, the same argument shows that for $\|\phi\|_{\sigma} \geqslant 1$ we also have a uniform lower bound on $|\phi|_{\sigma}(z)$ when $r(z)$ is greater than a fixed positive multiple of $\|\phi\|_{\sigma}^{1 / 2}$.

Thus to complete the argument it suffices to consider the case when $r(z)$ is small compared to $\|\phi\|_{\sigma}^{1 / 2}$. That is, we consider a point $z$ in a disk of radius $\epsilon\|\phi\|_{\sigma}^{1 / 2}$ about one of the zeros. Equivalently, if we let $\phi_{0}=\|\phi\|_{\sigma}^{-1} \phi$, then $z$ lies in a disk of $\phi_{0}$-radius $\epsilon$ about a zero of $\phi_{0}$. Assume that $\epsilon$ is small enough (depending on the ray) so that there is only one zero of $\phi_{0}$ in this disk, and that the disk is identified with $|\zeta|<R$ by a coordinate function $\zeta$ such that $\phi_{0}=\zeta \mathrm{d} \zeta^{2}$. We work in this coordinate system for the rest of the proof.

Write the Poincaré metric of $C$ on this disk as $\mathrm{e}^{2 \sigma}|\mathrm{d} \zeta|^{2}$. Then, using compactness of the unit ball in $\mathcal{B}$ again, we have $\mathrm{e}^{2 \sigma} \leqslant M$ for a uniform constant $M$.

The $\phi_{0}$-distance from 0 to $\zeta$ is proportional to $|\zeta|^{3 / 2}$, and thus the $\phi$-distance is proportional to $\|\phi\|_{\sigma}^{1 / 2}|\zeta|^{3 / 2}$, with universal constants in both cases. The hypothesis that $r(z) \geqslant 1$ therefore becomes $|\zeta(z)| \geqslant c\|\phi\|_{\sigma}^{-1 / 3}$ for a constant $c>0$. Using that $\phi=\|\phi\|_{\sigma} \zeta \mathrm{d} \zeta^{2}$, at such a point $z$ we have

$$
|\phi|_{\sigma}(z)=\mathrm{e}^{-2 \sigma(z)}\|\phi\|_{\sigma}|\zeta(z)| \geqslant M^{-1}\|\phi\|_{\sigma}\left(c\|\phi\|_{\sigma}^{-1 / 3}\right)=M^{-1} c\|\phi\|_{\sigma}^{2 / 3}
$$

Since we assumed $\|\phi\|_{\sigma} \geqslant 1$, this gives the desired lower bound with $\delta=M^{-1} c$.

\subsection{Exponential bounds.}

Proof of Theorem 8. Define

$$
f=F-\frac{1}{2} \frac{\dot{\phi}}{\phi}
$$

so that our goal is to give an exponentially decaying upper bound on $|f|$ at a point $z$. As in the proof of Theorem 5 we first choose an immersed coordinate chart $|\zeta|<r(z)$ where $\phi=\mathrm{d} \zeta^{2}$ and $z$ corresponds to $\zeta=0$.

Using (4.14), after a bit of algebra we find that in this coordinate system $f$ satisfies the equation

$$
(\Delta-16 \cosh (2 u)) f=-8 \frac{\dot{\phi}}{\phi} \sinh (2 u) .
$$

Let $\epsilon>0$, and restrict attention to the smaller disk $\Omega=\{|\zeta| \leqslant(1-\epsilon) r(z)\}$. Assume that $r(z)>\epsilon^{-1}$. Then all points of $\Omega$ are at distance at least 1 from $\phi^{-1}(0)$, and Lemma 10 gives

$$
\left|\frac{\dot{\phi}}{\phi}\right|=\frac{|\dot{\phi}|_{\sigma}}{|\phi|_{\sigma}} \leqslant \delta^{-1}\|\dot{\phi}\|_{\sigma}
$$


throughout $\Omega$. Now fix $\gamma<4$, let $b(\gamma)$ denote the constant from Theorem 5 , and assume that $r(z)>\epsilon^{-1} b(\gamma)$. Then Theorem 5 applies to $u$ at each point of $\Omega$, giving

$$
|u(\zeta)|<A(\gamma) \mathrm{e}^{-\gamma r(\zeta)} .
$$

Combining this with the bound (7.14) on $\frac{\dot{\phi}}{\phi}$, we find that the right hand side of (7.13) is bounded above by

$$
A^{\prime}(\gamma)\|\dot{\phi}\|_{\sigma} \mathrm{e}^{-\gamma r(\zeta)}
$$

for some constant $A^{\prime}(\gamma)$.

Let $\rho$ denote the function on $\Omega$ that gives the $\phi$-distance to $\partial \Omega$. Since $r(z)>\rho(z)$ we can replace $(7.16)$ by

$$
A^{\prime}(\gamma)\|\dot{\phi}\|_{\sigma} \mathrm{e}^{-\gamma \rho}
$$

When combined with the fact that $16 \cosh (2 u) \geqslant 16$, this exponential decay of the inhomogeneous term of (7.13) implies that the solution $f$ is also exponentially decaying; specifically, applying Theorem 3 we have for any $\gamma<4$

$$
|f| \leqslant K(\gamma)\left(M+A^{\prime \prime}(\gamma)\|\dot{\phi}\|_{\sigma}\right) \mathrm{e}^{-\gamma \rho}
$$

where $M=\sup _{\partial \Omega}|f|$. We have $|f| \leqslant|F|+\frac{1}{2}\left|\frac{\dot{\phi}}{\phi}\right|$, and therefore Lemma 9 and (7.14) give $M \leqslant\left(1+\delta^{-1}\right)\|\dot{\phi}\|_{\sigma}$. Substituting this value for $M$ into (7.18) and evaluating at $\zeta=0$ (i.e. at $z)$, where $\rho=(1-\epsilon) r(z)$, we obtain

$$
|f(z)| \leqslant A^{\prime \prime \prime}(\gamma)\|\dot{\phi}\|_{\sigma} \mathrm{e}^{-\gamma(1-\epsilon) r(z)}
$$

for a constant $A^{\prime \prime \prime}(\gamma)$ depending on the ray $\mathbb{R}_{+} \phi$. Since $\epsilon$ was arbitrary, this gives the desired bound.

\section{HOLOMORPHIC VARIATIONS}

In our analysis of $C_{\text {near }}$ we will exploit the following basic observation: if $D \subset C$ is a disk containing exactly one zero of $\phi$, then any holomorphic quadratic differential $\dot{\phi}$ on $D$ can be realized as $\dot{\phi}=\mathcal{L}_{X} \phi$ for some holomorphic vector field $X$ on $D$. This fact allows us to construct an explicit solution of the complex variation equation (4.14) on $D$, using the following:

Theorem 11. Given a quadratic differential $\phi=P(z) \mathrm{d} z^{2}$, solution $u$ of (4.3), and holomorphic vector field $X=\chi(z) \frac{\partial}{\partial z}$, let

$$
\psi_{X}=\mathcal{L}_{X} \phi=\left(\chi P_{z}+2 \chi_{z} P\right) \mathrm{d} z^{2}
$$

and define a complex scalar function $F_{X}$ by

$$
F_{X} \mathrm{e}^{2 u}|\mathrm{~d} z|^{2}=\mathcal{L}_{X}\left(\mathrm{e}^{2 u}|\mathrm{~d} z|^{2}\right)
$$

or equivalently in local coordinates

$$
F_{X}=\chi_{z}+2 \chi u_{z}
$$

Then $F=F_{X}$ satisfies the complex variation equation (4.14) with $\dot{\phi}=\psi_{X}$. 
Proof. We begin by noting that the self-duality equation (4.3) is natural with respect to biholomorphic maps, i.e. if $\Phi$ is such a map then the log density of the pullback metric $\Phi^{*}\left(\mathrm{e}^{2 u}|\mathrm{~d} z|^{2}\right)$ satisfies the equation for the pullback differential $\Phi^{*} \phi$. The real vector field $X+\bar{X}$ has a local flow which consists of holomorphic maps, and hence gives rise to a local 1parameter family of solutions for the corresponding family of pullback quadratic differentials. Taking the derivative of this family of solutions at $t=0$ we find that the Lie derivative of $\mathrm{e}^{2 u}|\mathrm{~d} z|^{2}$ with respect to $X+\bar{X}$ gives a solution of the variation equation (4.4) for

$$
\dot{\phi}=\mathcal{L}_{X+\bar{X}} \phi \text {. }
$$

Specifically, if we define $\dot{u}$ by

$$
\dot{u} \mathrm{e}^{2 u}|\mathrm{~d} z|^{2}=\mathcal{L}_{X+\bar{X}}\left(\mathrm{e}^{2 u}|\mathrm{~d} z|^{2}\right)
$$

then $\dot{u}$ and $\dot{\phi}$ satisfy (4.4). The expression (8.5) is equivalent to saying that $\dot{u}$ is the Riemannian divergence of the vector field $X+\bar{X}$ with respect to the metric $\mathrm{e}^{2 u}|\mathrm{~d} z|^{2}$.

Now, recall that (4.14) is equivalent to the separate equations (4.4) for $\dot{u}=\operatorname{Re}(F)$ and (4.12) for $v=-\operatorname{Im}(F)$, and that these two equations are related by the substitutions $\dot{u} \rightarrow-v$ and $\dot{\phi} \rightarrow \mathrm{i} \dot{\phi}$.

For a real tensor $T$ we have $\operatorname{Re}\left(\mathcal{L}_{X} T\right)=\mathcal{L}_{X+\bar{X}} T$, and hence $\operatorname{Re}\left(F_{X}\right)$ is exactly $\dot{u}$ as defined by (8.5), which we have seen satisfies (4.4) with $\dot{\phi}=\mathcal{L}_{X+\bar{X}} \phi$. Because $\phi$ is holomorphic we in fact have $\mathcal{L}_{X+\bar{X}} \phi=\mathcal{L}_{X} \phi=\psi_{X}$. Hence $\operatorname{Re}\left(F_{X}\right)$ satisfies the desired equation.

Because $\mathcal{L}_{\mathrm{i} X}=\mathrm{i} \mathcal{L}_{X}$ we have $-\operatorname{Im}\left(F_{X}\right)=\operatorname{Re}\left(F_{\mathrm{i} X}\right)$ which therefore satisfies (4.4) with $\dot{\phi}=\mathcal{L}_{\mathrm{i} X+i \bar{X}} \phi=\mathcal{L}_{\mathrm{i} X} \phi=\mathrm{i} \psi_{X}$. Using the substitutions noted above, this is equivalent to $\operatorname{Im}\left(F_{X}\right)$ satisfying $(4.12)$.

\section{EXACTNESS}

Given quadratic differentials $\phi$ and $\dot{\phi}$ on a compact Riemann surface $C$, recall that our ultimate goal is to bound the difference

$$
\Delta(\phi, \dot{\phi}):=g_{\phi}(\dot{\phi}, \dot{\phi})-g_{\phi}^{\mathrm{sf}}(\dot{\phi}, \dot{\phi})
$$

Though the integrals defining $g_{\phi}$ and $g_{\phi}^{\text {sf }}$ were previously written in terms of densities (scalar multiples of $\mathrm{d} x \mathrm{~d} y$ ), using the orientation of $C$ we can convert the integrand to a differential 2 -form which we denote by $\delta$. Also recall that this integrand depends on the density $u$ and complex function $F$, respectively satisfying (4.3) and (4.14). Explicitly, by taking the difference of the integral expressions (4.23)-(4.24) we find

$$
\delta(\phi, \dot{\phi}, u, F)=\left(4 \mathrm{e}^{-2 u}\left(|\dot{P}|^{2}-\operatorname{Re}(F P \dot{\bar{P}})\right)-2 \frac{|\dot{P}|^{2}}{|P|}\right) \mathrm{d} x \wedge \mathrm{d} y,
$$

where $\dot{\phi}=\dot{P} \mathrm{~d} z^{2}$ and as usual $\phi=P \mathrm{~d} z^{2}$. Thus if $u(\phi)$ and $F(\phi, \dot{\phi})$ denote the unique solutions to (4.3) and (4.14) on a compact Riemann surface $C$ for given $\phi$ and $\dot{\phi}$, then we have

$$
\Delta(\phi, \dot{\phi})=\int_{C} \delta(\phi, \dot{\phi}, u(\phi), F(\phi, \dot{\phi})) .
$$

As mentioned in $\S 1.2$, our technique for bounding the integral of $\delta(\phi, \dot{\phi}, u(\phi), F(\phi, \dot{\phi}))$ over the region $C_{\text {near }}$ near the zeros of $\phi$ involves approximating $\delta$ in that region by an exact form. The key to this approximation is that $\delta(\phi, \dot{\phi}, u, F)$ itself is exact whenever $\dot{\phi}$ and $F$ are obtained from $\phi$ and $u$ using a holomorphic vector field as in Theorem 11: 
Lemma 12. Let $\phi=P \mathrm{~d} z^{2}$ be a quadratic differential and $u$ a log density satisfying (4.3), both on a domain $U \subset \mathbb{C}$. Let $X=\chi \partial_{z}$ be a holomorphic vector field on $U$. Let $\dot{\phi}=\mathcal{L}_{X} \phi$ and $F=F_{X}$ as in Theorem 11. Then $\delta(\phi, \dot{\phi}, u, F)=\mathrm{d} \beta$, where

$$
\beta=\left(\mathrm{e}^{-2 u}-|P|^{-1}\right)\left(2|P|^{2} \star \mathrm{d}|\chi|^{2}+|\chi|^{2} \star \mathrm{d}|P|^{2}\right) .
$$

Proof. Substituting $\dot{\phi}=\mathcal{L}_{X} \phi$ as given by (8.1) and $F=F_{X}$ from (8.2) into (9.2), we obtain an explicit formula in terms of $\chi, P$, and $u$ :

$$
\begin{aligned}
\delta\left(\phi, \mathcal{L}_{\chi} \phi, u, F_{X}\right) & =\left(4 \mathrm { e } ^ { - 2 u } \left(\left|\chi P_{z}\right|^{2}+2\left|\chi_{z} P\right|^{2}+3 \operatorname{Re}\left(\chi \bar{\chi}_{\bar{z}} P_{z} \bar{P}\right)-2 \operatorname{Re}\left(\chi \bar{\chi} P \bar{P}_{\bar{z}} u_{z}\right)\right.\right. \\
- & \left.\left.4 \operatorname{Re}\left(\chi \bar{\chi}_{\bar{z}} P \bar{P} u_{z}\right)\right)-2 \frac{\left|\chi P_{z}\right|^{2}}{|P|}-8 \frac{\operatorname{Re}\left(\chi \bar{\chi}_{\bar{z}} \bar{P} P_{z}\right)}{|P|}-8\left|\chi_{z}\right|^{2}|P|\right) \mathrm{d} x \wedge \mathrm{d} y .
\end{aligned}
$$

Now we consider $\beta$. For a holomorphic function $f$, we have

$$
\star \mathrm{d}|f|^{2}=\star \mathrm{d}(f \bar{f})=\star\left(f_{z} \bar{f} \mathrm{~d} z+f \bar{f}_{\bar{z}} \mathrm{~d} \bar{z}\right)=-\mathrm{i}\left(f_{z} \bar{f} \mathrm{~d} z-f \bar{f}_{\bar{z}} \mathrm{~d} \bar{z}\right)=2 \operatorname{Im}\left(f_{z} \bar{f} \mathrm{~d} z\right) .
$$

Using this, we find that $\beta=2 \operatorname{Im}(\tilde{\beta})$ where

$$
\tilde{\beta}=\left(\mathrm{e}^{-2 u}-|P|^{-1}\right)\left(2|P|^{2} \chi_{z} \bar{\chi}+|\chi|^{2} P_{z} \bar{P}\right) \mathrm{d} z .
$$

It is then straightforward to calculate $\mathrm{d} \beta=2 \operatorname{Im}(\bar{\partial} \tilde{\beta})$ in terms of $P$ and $\chi$, and to verify that it is equal to (9.5); in the latter step, it is useful to recall $\operatorname{Im}(c \mathrm{~d} \bar{z} \wedge \mathrm{d} z)=2 \operatorname{Re}(c) \mathrm{d} x \wedge \mathrm{d} y$ for any complex scalar $c$. We omit the details of this lengthy but elementary calculation.

\section{Exponential ASYMPTOTICS}

In this section we prove Theorem 1 . To do so we return to considering a compact Riemann surface $C$ and the ray $\left\{\phi=t \phi_{0}\right\}_{t \in \mathbb{R}_{+}}$generated by $\phi_{0} \in \mathcal{B}^{\prime}$. Write $\phi=P \mathrm{~d} z^{2}$. Let $\dot{\phi}=$ $\dot{P} \mathrm{~d} z^{2} \in T_{\phi} \mathcal{B}^{\prime}=\mathcal{B}$. Fix some $\gamma<4$.

Let $z_{1}, \ldots, z_{n}$ denote the zeros of $\phi$, and let $D_{i}$ denote an open disk centered on $z_{i}$ of $|\phi|$-radius

$$
R=\frac{1}{2} M(\phi)=\frac{1}{2} t^{\frac{1}{2}} M\left(\phi_{0}\right)
$$

This is the largest $\phi$-radius for which the sets $D_{i}$ are disjoint, embedded disks. Note that $D_{i}$ can also be described as the disk about $z_{i}$ of $\left|\phi_{0}\right|$-radius $\frac{1}{2} M\left(\phi_{0}\right)$, and in particular the set $D_{i}$ is independent of $t$.

Since we are considering asymptotic statements as $t \rightarrow \infty$, and since by hypothesis $M\left(\phi_{0}\right)>0$, we may assume when necessary that $R$ is larger than any given constant.

Let $C_{\text {near }}=\bigcup_{i} D_{i}$ and $C_{\text {far }}=C \backslash C_{\text {near }}$. Then we have

$$
\Delta(\phi, \dot{\phi})=\int_{C_{\mathrm{far}}} \delta(\phi, \dot{\phi}, u(\phi), F(\phi, \dot{\phi}))+\sum_{i} \int_{D_{i}} \delta(\phi, \dot{\phi}, u(\phi), F(\phi, \dot{\phi})),
$$

and we will bound these terms separately.

The "far" region. For any $z \in C_{\text {far }}$ we have $r(z) \geqslant R$. Assume $R$ is large enough so that Theorem 5 and Theorem 8 apply. Then we have $u \approx \frac{1}{2} \log |P|$ and $F \approx \frac{1}{2} \frac{\dot{P}}{P}$ with respective errors bounded by $a(\gamma) \mathrm{e}^{-\gamma R}$ and $a(\gamma)\|\dot{\phi}\|_{\sigma} \mathrm{e}^{-\gamma R}$ for some constant $a(\gamma)$. If these approximate 
equalities were exact, then $\delta$ would vanish identically; that is, by direct substitution into the definition (9.2), we find that

$$
\delta\left(\phi, \dot{\phi}, \frac{1}{2} \log |P|, \frac{1}{2} \frac{\dot{P}}{P}\right)=0 .
$$

To handle the situation at hand, we will strengthen this to show that $\delta$ is pointwise small when $u$ and $F$ are only near $\frac{1}{2} \log |P|$ and $\frac{1}{2} \frac{\dot{P}}{P}$ (respectively).

Again by substitution into (9.2), we find that for any scalar functions $w$ and $\mu$ we have

$$
\delta\left(\phi, \dot{\phi}, \frac{1}{2} \log |P|+w, \frac{1}{2} \frac{\dot{P}}{P}+\mu\right)=\left(2 \frac{|\dot{P}|^{2}}{|P|}\left(\mathrm{e}^{-2 w}-1\right)-4 \frac{\mathrm{e}^{-2 w}}{|P|} \operatorname{Re}(P \overline{\dot{P}} \mu)\right) \mathrm{d} x \wedge \mathrm{d} y \text {. }
$$

Now assume that $|w|<1$, so that

$$
2\left|\mathrm{e}^{-2 w}-1\right| \leqslant c|w|
$$

and

$$
\left.\left|4 \mathrm{e}^{-2 w}\right| P\right|^{-1} \operatorname{Re}(P \overline{\dot{P}} \mu)|\leqslant c| \dot{P}|| \mu \mid
$$

for a constant $c>0$. Using these estimates with (10.4) gives

$$
\left|\frac{\delta\left(\phi, \dot{\phi}, \frac{1}{2} \log |P|+w, \frac{1}{2} \frac{\dot{P}}{P}+\mu\right)}{\mathrm{d} x \wedge \mathrm{d} y}\right| \leqslant c\left(\frac{|\dot{P}|^{2}}{|P|}|w|+|\dot{P}||\mu|\right) .
$$

If we furthermore assume $R>1$, then Lemma 10 applies to $\phi$ throughout $C_{\mathrm{far}}$, giving a uniform lower bound on $\mathrm{e}^{-2 \sigma}|P|$. Substituting this into the previous bound, we can now bound $\delta$ relative to the hyperbolic area form as follows:

$$
\left|\delta\left(\phi, \dot{\phi}, \frac{1}{2} \log |P|+w, \frac{1}{2} \frac{\dot{P}}{P}+\mu\right)\right| \leqslant c\left(|\dot{\phi}|_{\sigma}^{2}|w|+|\dot{\phi}|_{\sigma}|\mu|\right) \mathrm{e}^{2 \sigma}|\mathrm{d} z|^{2} .
$$

Here $c$ is a constant, but not the same constant as in (10.7). We already observed that on $C_{\text {far }}$ the integrand $\delta(\phi, \dot{\phi}, u(\phi), F(\phi, \dot{\phi}))$ has the form (10.4) with $|w| \leqslant a(\gamma) \mathrm{e}^{-\gamma R}$ and $|\mu| \leqslant a(\gamma)\|\dot{\phi}\|_{\sigma} \mathrm{e}^{-\gamma R}$. Thus

$$
|\delta(\phi, \dot{\phi}, u(\phi), F(\phi, \dot{\phi}))| \leqslant c^{\prime}(\gamma)|\phi|_{\sigma}^{2} \mathrm{e}^{-\gamma R} \mathrm{e}^{2 \sigma}|\mathrm{d} z|^{2} \text { on } C_{\text {far }},
$$

for a constant $c^{\prime}(\gamma)$. Integrating (10.9), and using that the $\sigma$-area of $C_{\text {far }}$ is bounded and $|\dot{\phi}|_{\sigma} \leqslant\|\dot{\phi}\|_{\sigma}$, we obtain

$$
\int_{C_{\mathrm{far}}} \delta(\phi, \dot{\phi}, u(\phi), F(\phi, \dot{\phi}))=O\left(\|\dot{\phi}\|_{\sigma}^{2} \mathrm{e}^{-\gamma R}\right)
$$

with the implicit constant depending only on $c^{\prime}(\gamma)$ from (10.9).

The "near" region. Next we consider the integral over one of the disks $D_{i}$ in $(10.2)$. Identify $D_{i}$ with a disk $\{|z|<R\}$ in $\mathbb{C}$, using a coordinate $z$ in which $\left.\phi\right|_{D_{i}}=z \mathrm{~d} z^{2}$.

On $D_{i}$ there is a unique holomorphic vector field $X=\chi \partial_{z}$ such that $\dot{\phi}=\mathcal{L}_{X} \phi=\mathcal{L}_{X}\left(z \mathrm{~d} z^{2}\right)$; explicitly, if we write

$$
\dot{\phi}=\sum_{n} a_{n} z^{n} \mathrm{~d} z^{2}
$$


then

$$
\chi=\sum_{n} \frac{a_{n}}{2 n+1} z^{n}
$$

By Theorem 11, the associated function $F_{X}$ defined by $F_{X} \mathrm{e}^{2 u(\phi)}|\mathrm{d} z|^{2}=\mathcal{L}_{X}\left(\mathrm{e}^{2 u(\phi)}|\mathrm{d} z|^{2}\right)$ satisfies (4.14) on $D_{i}$, which is the same equation satisfied by $F(\phi, \dot{\phi})$. We will show that $F_{X}$ and $F(\phi, \dot{\phi})$ are in fact exponentially close on $D_{i}$.

First we consider the restrictions of these functions to $\partial D_{i}$, which is far from the zeros of $\phi$, allowing the estimates of the previous sections to be applied. By Theorem 8 we have

$$
\left|F(\phi, \dot{\phi})-\frac{1}{2} \frac{\dot{P}}{P}\right|=O\left(\|\dot{\phi}\|_{\sigma} \mathrm{e}^{-\gamma R}\right) \text { on } \partial D_{i} .
$$

Turning to $F_{X}=\chi_{z}+2 \chi u_{z}$, note that the equation $\dot{\phi}=\mathcal{L}_{X} \phi$ gives

$$
\chi_{z}=\frac{\dot{P}-\chi P_{z}}{2 P}=\frac{\dot{P}}{2 P}-\chi \partial_{z}(\log |P|)
$$

and thus

$$
F_{X}=\chi_{z}+2 \chi u_{z}=\frac{\dot{P}}{2 P}+2 \chi \partial_{z}\left(u-\frac{1}{2} \log |P|\right) .
$$

By the $C^{1}$ bound from Theorem 5 we have

$$
\partial_{z}\left(u-\frac{1}{2} \log |P|\right)=O\left(\mathrm{e}^{-\gamma R}\right) \text { on } \partial D_{i} .
$$

Next we need a bound on $|\chi|$ on $\partial D_{i}$. For this, note that for any $z \in D_{i}, \chi(z)$ depends linearly on $\dot{\phi}$, and scales as $t^{-\frac{2}{3}}$. Thus, for $t>1$ we have an estimate $|\chi(z)|<c(z)\|\dot{\phi}\|_{\sigma}$ for some $c(z)$, and since the closure of $D_{i}$ is compact we can take this constant to be independent of $z$, i.e. on $D_{i}$ we have

$$
|\chi|=O\left(\|\dot{\phi}\|_{\sigma}\right) \text {. }
$$

Now combining (10.16) and (10.17), we get

$$
\left|F_{X}-\frac{1}{2} \frac{\dot{P}}{P}\right|=O\left(\|\dot{\phi}\|_{\sigma} \mathrm{e}^{-\gamma R}\right) \text { on } \partial D_{i} .
$$

Then by (10.13) and (10.18) we find that the function $\mu: D_{i} \rightarrow \mathbb{C}$ defined by

$$
\mu=F_{X}-F(\phi, \dot{\phi})
$$

satisfies

$$
\mu=O\left(\|\dot{\phi}\|_{\sigma} \mathrm{e}^{-\gamma R}\right) \text { on } \partial D_{i}
$$

Because $F_{X}$ and $F(\phi, \dot{\phi})$ both satisfy the linear inhomogeneous equation (4.14), their difference $\mu$ satisfies the associated homogeneous equation, which has the form $(\Delta-k) \mu=0$ for an everywhere positive function $k$ (compare (7.5)); this implies that $|\mu|$ has no interior maximum. Thus $|\mu|$ achieves its maximum on $\partial D_{i}$, and (10.20) gives

$$
\mu=O\left(\|\dot{\phi}\|_{\sigma} \mathrm{e}^{-\gamma R}\right) \text { on } D_{i} .
$$


Next we use this estimate on $\mu$ to estimate the integral of $\delta(\phi, \dot{\phi}, u(\phi), F(\phi, \dot{\phi}))$ over $D_{i}$. We have

$$
\begin{aligned}
\delta(\phi, \dot{\phi}, u(\phi), F(\phi, \dot{\phi})) & =\delta\left(\phi, \dot{\phi}, u(\phi), F_{X}+\mu\right) \\
& =\delta\left(\phi, \dot{\phi}, u(\phi), F_{X}\right)+4 \mathrm{e}^{-2 u(\phi)} \operatorname{Re}(\mu P \dot{P}) \mathrm{d} x \wedge \mathrm{d} y .
\end{aligned}
$$

Since $\mathrm{e}^{-2 u(\phi)}|P| \leqslant 1$ (by Lemma 6) we have

$$
\left|4 \mathrm{e}^{-2 u(\phi)} \operatorname{Re}(\mu P \dot{\dot{P}})\right| \leqslant 4|\mu||\dot{P}|
$$

and the bound (10.21) gives

$$
\int_{D_{i}} 4 \mathrm{e}^{-2 u(\phi)} \operatorname{Re}(\mu P \overline{\dot{P}}) \mathrm{d} x \wedge \mathrm{d} y=O\left(\|\dot{\phi}\|_{\sigma}^{2} \mathrm{e}^{-\gamma R}\right) .
$$

Considering the other term on the right hand side of (10.22), by Lemma 12 the form $\delta\left(\phi, \dot{\phi}, u, F_{X}\right)$ is exact, so we can use Stokes's theorem to reduce to a boundary term:

$$
\int_{D_{i}} \delta\left(\phi, \dot{\phi}, u, F_{X}\right)=\int_{\partial D_{i}}\left(\mathrm{e}^{-2 u}-|P|^{-1}\right)\left(2|P|^{2} \star \mathrm{d}|\chi|^{2}+|\chi|^{2} \star \mathrm{d}|P|^{2}\right) .
$$

It just remains to show that this boundary term is exponentially small. Fix some $\gamma^{\prime}$ with $\gamma<\gamma^{\prime}<4$. By Theorem 5, we have $\left(\mathrm{e}^{-2 u}-|P|^{-1}\right)=O\left(\mathrm{e}^{-\gamma^{\prime} R}\right)$ on $\partial D_{i}$. Next, using the estimate (10.17), the fact that $P$ scales as $t$, and the fact that the coordinate radius of $D_{i}$ scales as $t^{1 / 3}$, we have

$$
\int_{\partial D_{i}}\left(2|P|^{2} \star \mathrm{d}|\chi|^{2}+|\chi|^{2} \star \mathrm{d}|P|^{2}\right)=O\left(t^{\frac{7}{3}}\|\dot{\phi}\|_{\sigma}^{2}\right) .
$$

Using this in (10.25) gives

$$
\int_{D_{i}} \delta\left(\phi, \dot{\phi}, u, F_{X}\right)=O\left(t^{\frac{7}{3}}\|\dot{\phi}\|_{\sigma}^{2} \mathrm{e}^{-\gamma^{\prime} R}\right)=O\left(\|\dot{\phi}\|_{\sigma}^{2} \mathrm{e}^{-\gamma R}\right)
$$

where in the last equality we use the fact that $R \rightarrow \infty$ as $t \rightarrow \infty$ by (10.1).

Now we have bounded the integrals of both terms in (10.22); combining these bounds we conclude

$$
\int_{D_{i}} \delta(\phi, \dot{\phi}, u(\phi), F(\phi, \dot{\phi}))=O\left(\|\dot{\phi}\|_{\sigma}^{2} \mathrm{e}^{-\gamma R}\right) .
$$

Summing up. Finally, substituting the far and near bounds ((10.10) and (10.28)) into (10.2), we obtain

$$
\Delta(\phi, \dot{\phi})=O\left(\|\dot{\phi}\|_{\sigma}^{2} \mathrm{e}^{-\gamma R}\right)
$$

Using (10.1), and that $\gamma<4$ was arbitrary, we obtain the exponential bound from Theorem 1 by taking $\gamma=8 \alpha / M\left(\phi_{0}\right)$.

Finally, we consider the contributions to the multiplicative constant in (10.29). We have seen that the individual exponential estimates in the components of $C_{\text {near }}$ depend only on $\gamma$. The number of such components is linear in the genus of $C$. The estimate in $C_{\text {far }}$ obtained above is also linear in the hyperbolic area of $C$, or equivalently in the genus. Overall we find the multiplicative constant in the final estimate depends on $\gamma$ and the topology of $C$, or equivalently, on $\alpha, M\left(\phi_{0}\right)$, and the genus. This completes the proof of Theorem 1 . 


\section{REFERENCES}

[1] M. Abramowitz and I. Stegun. Handbook of mathematical functions with formulas, graphs, and mathematical tables. Dover Publications, New York, 1965.

[2] L. V. Ahlfors. Conformal invariants: topics in geometric function theory. McGraw-Hill, New York, 1973.

[3] D. Dumas and M. Wolf. Polynomial cubic differentials and convex polygons in the projective plane. Geom. Funct. Anal., 25(6):1734-1798, 2015.

[4] D. Dumas and M. Wolf. blaschke: Compute polygonal affine spheres corresponding to complex polynomials. https://github.com/daviddumas/blaschke, 2014.

[5] D. S. Freed. Special Kähler manifolds. Commun. Math. Phys., 203:31-52, 1999, arXiv: hep-th/9712042.

[6] D. Gaiotto, G. W. Moore, and A. Neitzke. Four-dimensional wall-crossing via three-dimensional field theory. Commun. Math. Phys., 299:163-224, 2010, arXiv:0807.4723.

[7] D. Gaiotto, G. W. Moore, and A. Neitzke. Spectral networks. Annales Henri Poincaré, 14(7):1643-1731, 11 2013, arXiv: 1204.4824.

[8] D. Gaiotto, G. W. Moore, and A. Neitzke. Wall-crossing, Hitchin systems, and the WKB approximation. Adv. Math., 234:239-403, 2013, arXiv:0907.3987.

[9] N. J. Hitchin. The self-duality equations on a Riemann surface. Proc. London Math. Soc. (3), 55(1):59$126,1987$.

[10] N. J. Hitchin. Stable bundles and integrable systems. Duke Math. J., 54(1):91-114, 1987.

[11] R. Mazzeo, J. Swoboda, H. Weiss, and F. Witt. Asymptotic geometry of the Hitchin metric. arXiv: $1709.03433 \mathrm{v} 1$.

[12] Y. N. Minsky. Harmonic maps, length, and energy in Teichmüller space. J. Differential Geom., $35(1): 151-217,1992$.

[13] A. Neitzke. Notes on a new construction of hyperkahler metrics. In Homological Mirror Symmetry and Tropical Geometry, volume 15 of Lect. Notes Unione Mat. Ital., pages 351-375. Springer, Cham, 2014, arXiv:1308.2198.

[14] M. Wolf. High energy degeneration of harmonic maps between surfaces and rays in Teichmüller space. Topology, 30(4):517-540, 1991.

Department of Mathematics, Statistics, and Computer Science

University of Illinois at Chicago

Chicago, IL

david@dumas.io

Department of Mathematics

University of Texas at Austin

Austin, TX

neitzke@math.utexas.edu 